\title{
Conversaciones en torno de la narrativa de experiencia y la creatividad docente en el currículum
}

\section{Daniel Hugo Suárez}

Universidad de Buenos Aires, Facultad de Filosofía y Letras, Argentina

mail: dhsuarezQfilo.uba.ar

ORCID: https://0000-0002-2438-7145

\author{
Valeria Marta Metzdorff \\ Escuela N¹7, Distrito Escolar 9no., Ciudad Autónoma de \\ Buenos Aires, Argentina \\ mail: valeria.metzdorffabue.edu.ar \\ ORCID: https://0000-0002-2824-2349
}

\section{RESUMEN}

El artículo recrea un momento de la conversación siempre abierta entre una tesista y su director a partir de la invitación de éste a pensar en la creatividad docente en el currículum. Al plantearse como reflexión, la propuesta desata una breve presentación del vínculo que cada uno ha venido construyendo con la idea del "currículum como creación" o bien de "la creación en el currículum" desde su propia trayectoria intelectual y profesional. En el intercambio de relatos, reflexiones y argumentos se despliegan la perspectiva, la narración y los indicios de la creatividad pedagógica de una maestra de escuela primaria y de un investigador de la educación. Ella se expresa desde interrogantes, hallazgos y construcciones narrativas respecto de su "vuelta a la escuela", luego de cierto recorrido profesional por fuera de la enseñanza primaria. Por su parte, el investigador, narra sus reflexiones sobre la creatividad pedagógica en su recorrido por algunos debates curriculares y experiencias de investigación para dar cuenta de la gesta de una posición de repliegue, con la que interpela a su interlocutora, desde sus aproximaciones sucesivas hasta el abandono de la "retórica del currículum" como marco interpretativo de la productividad de los enseñantes. Del intercambio final emergen algunas pistas acerca de las potencialidades y los límites de las narrativas pedagógicas para dar cuenta de la forma en que el currículum puede ser comprendido como tradición selectiva y consensuada sobre la transmisión cultural y, al mismo tiempo, como territorio para disponer públicamente la experiencia creativa de los docentes en las escuelas.

Palabras Clave: Creatividad docente, currículum, relato de experiencia, experiencia pedagógica, conversación pedagógica.

\section{Conversations around experiential storytelling and teacher creativity in the curriculum}

\section{ABSTRACT}

This article reproduces the conversation between a PhD candidate and her supervisor as the latter invites her to reflect on the teacher's creativity in relation to the curriculum.

This reflection prompts a brief presentation on each of their own perspectives on the idea of "curriculum as creation" and "the creation of the curriculum", based on their own intellectual and professional experiences. In the exchange of stories, reflections, and arguments proposed in the article, the perspective, narration, and indications of the pedagogical creativity of a primary school teacher and a researcher on education unfold.

The PhD candidate contributes with questions, findings, and narrative constructions regarding her "return to school", after a certain professional journey outside primary education. On the other hand, the researcher narrates his reflections about pedagogical creativity alluding to some curriculum debates and research experiences, in order to interpellate the PhD candidate from her successive approaches to her abandonment of the "rhetoric of the curriculum" as an interpretative framework for the productivity of teachers. From the conversation between the two emerge some clues about the potentialities and limits of pedagogical narratives to account for the way in which the curriculum can be understood as a selective and consensual tradition of cultural transmission and, at the same time, as a territory for the creative experience of teachers in schools.

Keywords: Teacher creativity, curriculum, experience story, pedagogical experience, pedagogical conversation.

ISSN: 0210-2773

DOI: https://doi.org/10.17811/rifie.50.3.2021.713-720 


\section{Un ensayo narrativo y en conversación}

Este ensayo narrativo y en diálogo es el resultado de una apuesta autobiográfica y conversacional para reescribir y repensar la creatividad pedagógica de los docentes en la escuela y en el currículum, en tres movimientos. Cada uno es una oportunidad para explorar un poco más descentrados el juego de las narrativas de sí, las experiencias vividas, los saberes pedagógicos y las obras de enseñanza (Alliaud, 2011, 2017) como creatividad docente y como investigación pedagógica, esto es, como inevitable exploración e innovación semántica propias del nombrar y renombrar, interpretar y recrear la experiencia escolar, el territorio del currículum y la pedagogía.

Partimos de la intención de recrear una conversación entre nosotros, los autores, una tesista y su director, a partir de la invitación de éste a pensar en la creatividad docente en el currículum. Al plantearse como reflexión, la propuesta desata una breve presentación del vínculo que cada uno ha venido construyendo con la idea del "currículum como creación" o bien de "la creación en el currículum" desde su propia trayectoria intelectual y profesional. En el intercambio de relatos, reflexiones y argumentos se despliega la perspectiva, la narración y los indicios de la creatividad pedagógica de una maestra de escuela primaria y de un investigador de la educación. Ella se expresa desde interrogantes, hallazgos y construcciones narrativas respecto de su "vuelta a la escuela", luego de cierto recorrido profesional por fuera de la enseñanza primaria. El, por su parte, narra sus reflexiones sobre la creatividad pedagógica en su recorrido por algunos debates curriculares y experiencias de investigación para dar cuenta de una posición de repliegue, con la que interpela a su interlocutora, desde sus aproximaciones sucesivas hasta el abandono de la "retórica del currículum" como marco interpretativo de la productividad de los enseñantes. Del intercambio final emergen algunas pistas acerca de las potencialidades y los límites de las narrativas pedagógicas para dar cuenta de la forma en que el currículum puede ser comprendido como tradición selectiva y consensuada sobre la transmisión cultural y, al mismo tiempo, como territorio para disponer públicamente la experiencia creativa de los docentes en las escuelas.

La puesta en escritura de una conversación requirió de una construcción que combinara elementos de conversaciones reales, con fragmentos agregados, referidos a aquellos datos, experiencias $\mathrm{u}$ opiniones que los que conversamos ya conocíamos del otro pero que aquí debieron explicitarse para los lectores. A su vez, dado que nuestras comprensiones se sustentan en las propias trayectorias profesionales, de formación y de investigación, constatamos la pertinencia de elaborar relatos para argumentar en torno de lo que cada uno de nosotros veníamos entendiendo como creatividad en el ámbito curricular. Esto nos llevó a configurar los tres movimientos del texto: dos relatos autobiográficos dirigidos, cada uno, al otro autor y un cierre de intercambio del que emergieron nuevos sentidos, surgidos del pensar de a dos acerca de aquellas comprensiones vividas por cada uno. El intercambio final muestra de qué manera un proceso de escritura en forma narrativa y conversacional resultó ser una experiencia enriquecedora de la que los autores nos retiramos distintos de cómo ingresamos. Así, sostuvimos la intención de explorar el potencial de la narración como forma discursiva para la construcción de saber y de la conversación en torno de relatos como dispositivo privilegiado para profundizar y elaborar nuevas comprensiones de los mundos que habitamos. $\mathrm{Y}$ particularmente en esta ocasión, lo hicimos desde la inspiración, aperturas, continuidades y exclusiones que nos plantea la metáfora del currículum como creación.

\section{La "vuelta a la escuela" como territorio de creatividad profesional}

En general, cuando me preguntan sobre mis prácticas de aula me siento compelida a elaborar un discurso expositivo que reproduzca, en primera persona, los textos curriculares o didácticos leídos en tercera. Me refiero a un constructo narrativo que manifieste mi asimilación de lo prescripto y que, a la medida del interlocutor, muestre u oculte lo que corresponda de acuerdo con, por definirlas de alguna manera, las prescripciones y prioridades curriculares y políticas del momento.

Como sabés, no siempre fui maestra. Cuando obtuve el título de Licenciada (en Ciencias de la Educación) logré ingresar a un órgano de evaluación universitaria en el que tenía que identificar en qué aspectos, las instituciones, los docentes y los alumnos, no cumplían a pie juntillas con las prescripciones y prioridades curriculares y políticas del momento.

Por ese bucle biográfico, tal vez te parezca natural que habiendo ocupado el rol policial unos quince años, haya caído en mi estado actual de prudencia e inclinación a la construcción discursiva cada vez que me preguntan sobre mis prácticas. Pero no es tan así.

Tu invitación a pensar en mis prácticas pedagógicas y la creatividad me encuentra en pleno proceso de tesis sobre mi vuelta a la escuela, al amparo de tu dirección. Es una tesis sostenida por una historia profesional e intelectual. Por eso, creo que debo responderte de una manera distinta, recurriendo al lenguaje de la narración, desde mi lugar de tesista invitada a la introspección sobre sus prácticas de maestra, en vez del de maestra interpelada por la prescripción curricular.

En realidad, desde mi formación docente y pedagógica diferentes lecturas y enseñanzas me mostraron las tensiones entre currículum y maestros; currículum y enseñanza, ámbitos de teoría y planificación y ámbitos de práctica y ejecución.

Imagino un pasado mítico, que seguramente opera desde las representaciones, en el que maestro y currículum no se distinguían, el maestro era el portador del saber y nadie le preguntaría sobre sus prácticas. Una imagen hoy inaudita y autoritaria del saber identificado masivamente con la figura del docente. Ese momento fundante de la identidad de un oficio, aunque tal vez no sea más que una fantasía, sigue teniendo su eficacia simbólica. Posiblemente por ella, hayamos vivido nuestra formación y nuestra práctica con cierto temor de sentirnos dueños del saber y de dejar alguna marca irreparable en nuestros alumnos (Alliaud, 2004) y, probablemente para evitarlo, deleguemos esa potestad en los especialistas, responsables de indicarnos cómo leer el documento curricular, es decir, cómo abordar el saber a enseñar.

Por eso, yo diría que la práctica, a falta de un especialista de bolsillo, siempre tiene lugar en cierto ámbito neblinoso de lo sabido, la duda y el deseo como condiciones de la creación. La práctica docente supone una lectura del documento curricular que, como toda práctica de lectura, se realiza desde una disposición subjetiva y situada y unas categorías y oposiciones que siempre son personales, relacionales, singulares y circunstanciales. En esta línea, hasta podría decirte que cualquier práctica normada expresa una traición a la norma objetiva porque toda lectura supone una "traición al texto", según aquello que nos contabas del significado "que se realiza en la lectura" en la inmanencia del texto, de acuerdo con la hermenéutica de Ricoeur (2004). Por eso, puede haber una variedad importante de prácticas plausibles y no plausibles en respuesta a cada prescripción positiva del currículum.

Cuando me preguntás acerca de mis prácticas se interpone mi pregunta sobre tu opinión respecto del diseño curricular y, 
a partir de ella, puedo contarte la misma clase escolar de varias maneras distintas. Como en esa frase atribuida a Groucho Marx sobre los principios.

Desde mis tiempos de formación, aprendí que el sentido común no era buen consejero para pensar la enseñanza y que esta debía someterse a la vigilancia epistemológica a fin de que la supuesta transposición didáctica se operara con arreglo a la estructura de la disciplina de origen $y$, a su vez, con pertinencia y relevancia suficiente como para justificar su enseñanza en el nivel primario (Camilloni, 1996; Litwin, 1997). El saber del especialista de la didáctica específica viene siendo el oráculo a consultar para no caer, como advierte Camilloni, en las consabidas "creaciones didácticas", en las que creaciones lleva un signo negativo. Entre ellas podemos mencionar varias creaciones famosas como las tablas de multiplicar, las lecturas sin sentido "Susana sala la masa", las fracciones propias, impropias o aparentes, el mapa de la Argentina sin sur o el modelo atómico parecido al sistema solar o el que se hace con borlas de plastilina.

Sin embargo, si bien son importantes estas advertencias, es cierto que hay un saber docente que calla frente a las propuestas de los especialistas de las didácticas disciplinares. Davini (2014) dejó plasmada en su último libro, cierta preocupación por el concepto mismo de transposición didáctica y la apariencia de inmutables y resueltas que nos sugiere respecto de las disciplinas escolares. Ella señalaba una orientación hacia el academicismo en las nuevas tendencias didácticas y advertía cierta desconfianza hacia el saber pedagógico de los docentes, consolidada por la reforma de los 90 y una serie continuada de programas de capacitación y evaluación de la enseñanza que se extiende hasta el presente (Suárez \& Metzdorff, 2018).

Como acompañante de residentes de primaria, percibí, en ocasiones, un desencuentro entre las propuestas de los especialistas y su pertinencia en las aulas con grupos escolares "reales". Pero atentos a la vigilancia epistemológica, habíamos ideado un dispositivo de acompañamiento que daba al especialista el rol de orientar la planificación y aprobar su implementación. Después de muchas reuniones en las que todos los profesores parecíamos estar de acuerdo, pero ninguno declinaba su posición, la solución fue llegando naturalmente a medida que los profesores de las didácticas específicas tomaron licencias para trabajar en editoriales, universidades y organismos de diseño curricular y sus cargos fueron cubiertos por otros que tenían, además de antecedentes en una didáctica específica, formación y experiencia como maestros de primaria. Con ese sólo criterio de selección pudo resolverse un debate improductivo entre miradas del aula escolar difíciles de integrar, significatividad lógica y psicológica versus sentido de la actividad, alumno ideal versus alumnos y grupos cualquiera.

Este año, me sentí muy halagada cuando me asignaron primer grado. Lo sentí como el desafío más hermoso de mi vuelta a la escuela. El tema del regreso a lo que fue descartado antes, viene siendo el aspecto de mi experiencia que más quisiera profundizar. El hecho de haber percibido, en determinado momento de mi carrera en la burocracia ministerial, la complejidad, riqueza y autonomía profesional del maestro, fue una epifanía probablemente estimulada por mis incursiones en diferentes escuelas para acompañar a las residentes. De pronto, la docencia abandonada para ascender a otros niveles del sistema apareció en mi universo como trabajo al que es conveniente llegar con cierta experiencia de vida, con algunos aprendizajes previos, cuanto más y más variados, mejor: haber recorrido el país, conocer los paisajes, las vidas, haber hecho estudios de posgrado, tener dos hijas, tocar la guitarra, saber bailar un gato, una chacarera o un tango, todo sumaba para una mayor valoración y disfrute de la docencia. Lo descartado entonces se convirtió en lo anhelado, en la meta reservada a quienes tienen experiencias para compartir. Así que emprendí el camino que me trajo hasta aquí. Desde entonces trabajé con diferentes edades durante un poco más de dos años, pero haber sido elegida para ocuparme de los chicos que llegan de la escuela infantil, fue todo un reconocimiento a $\mathrm{mi}$ esfuerzo por saldar tantos años de distancia. Creo que trabajar con los chicos que ingresan a la primaria es pensar la transmisión como una trama sin predominio de ningún hilo. Ahí van, con la misma prioridad, sentarse bien, aprender a contar, a sumar y a restar, cuidar por sí mismos sus cosas, usar los útiles sin desparramarlos ni morderlos, manejar solos la tijera y el pegamento sin jugar a la peluquería ni al gel para el cabello, tomar agua sin regar la cartuchera ajena, concentrarse, leer, todo en un escenario nuevo para los niños: el de la escuela primaria. Entre las rutinas y lo nuevo de cada día, hay que tejer esa trama para cada encuentro con los chicos. Creo que lo que más me fascina del trabajo en los primeros años de la escuela primaria, es que allí tiene lugar la construcción de los saberes del alumno, y por eso es crucial esa apertura al encuentro en el aula, estar disponible afectiva y mentalmente a compartir el tiempo y el lugar. Las maneras de presentar los contenidos son una forma más de promover ese encuentro.

Ese trabajo siempre requiere de varias lecturas del diseño curricular y de los documentos de desarrollo curricular. En lo que refiere a la lectoescritura, la producción didáctica para el área de Prácticas del Lenguaje es muy abundante. Queda claro que si, como explica Ferreiro (1997), el aprendizaje de la lectoescritura puede ser concebido como adquisición de un código o como construcción de un sistema de representación, el énfasis de nuestro diseño curricular está puesto en la segunda acepción. La lectoescritura se practica siempre en el marco de actividades con sentido social, con enfoque global, en el ambiente alfabetizador del aula, rico en agendas, carteles, listas de cuentos, nombres, registros de asistencia, calendarios, memos, etc. Esto no es novedad para ningún maestro de primero. Pero tal vez tampoco sea novedad para ellos y sí lo sea para mí, el hecho de que no haya en el diseño ni en los materiales curriculares ninguna mención a un trabajo explícito con las letras o con grupos de letras. Busqué en vano una línea que dijera "vocales", o M, L, S y T, las que suelen trabajarse primero en los gabinetes psicopedagógicos. Según perspectivas más analíticas y afines a la concepción del aprendizaje como adquisición de un código, esto se debe a la nitidez sonora de esas consonantes. Sin embargo, las propuestas didácticas de muchas editoriales brindan materiales que trabajan de esa manera. Y, ante la pregunta a mis colegas, la respuesta siempre me fue dada como opinión o decisión marginal: "Hay que ir mezclando".

Al parecer, me dije al comenzar el año, uno debería trabajar de manera global, recurriendo al mismo tiempo a las letras del nombre propio como a las palabras parecidas para activar lo que se ha denominado "conciencia fonológica" (mucho antes del boom de las neurociencias). Eso significa trabajar desde el primer día con todas las letras sobre la mesa, expuestas en la pared y usadas en todos los carteles. Pero, además, cada tanto, supuse, uno debería detener la marcha para reflexionar sobre algunas letras en particular, partiendo de las que tienen sonido más fácil de reconocer o se usan siempre de la misma manera, hasta llegar a las que pueden leerse de diferente forma o confundirse entre sí como la C, Q, K, V, B, entre otras. Este trabajo siempre debería derivar de alguna palabra importante de un cuento, una canción o de cualquier intercambio en torno de un texto literario, informativo o instructivo. Consistiría en combinar las letras que se están presentando con las vocales que se identifican en las pri- 
meras semanas de clase, formando sílabas simples que permitan hacer combinaciones para formar palabras. Entonces, a partir de un repertorio de sílabas simples, los alumnos podrían formar palabras, facilitando el pasaje de la etapa silábica a la etapa alfabética. Tales eran mis conjeturas.

De la letra del diseño curricular y sus materiales de desarrollo uno podría desprender que este aprendizaje también se alcanzaría siguiendo únicamente el enfoque global, como consecuencia de participar en muchas situaciones de lectura y escritura incorporando las reflexiones para adquirir conciencia fonológica en torno de las variaciones mínimas entre palabras parecidas: tela, tema, tala, toma... Pero ¡cómo cuesta! Los materiales de desarrollo curricular abundan en ejemplos de conversaciones riquísimas entre alumno y maestro como si ambos se encontrasen solos con una hoja y un lápiz o una pizarra y marcador borrable haciendo múltiples ensayos, escuchando y hablando en un ambiente sereno y silencioso. La maestra le muestra su escrito y lo lee en voz alta, el alumno reconoce el error en lo que escucha, pero no logra verlo en su escrito, la maestra cubre parte de la palabra y le lee la otra parte, la que tiene el error, el alumno propone la solución, escribe la letra faltante, pero en cualquier parte, la maestra vuelve a leerle lo que puso. Mientras tanto, los 22 compañeritos continúan atentos a sus escritos y en silencio como estudiantes universitarios.

Por mis lecturas de verano, había llegado a la conclusión de que hay autores (Pujato, 2009) que sostienen que el sistema de lectoescritura es ambas cosas, un sistema de representación al que se accede de manera pragmática y también un código con convenciones que se adquiere con ejercitación. Ambas perspectivas se han traducido en metodologías en disputa desde el siglo XIX (Braslavsky 2014 [1962]; Cucuzza y Spregelburd, 2012) y de lo que se trata es que el trabajo de aprendizaje no haga énfasis en la segunda acepción para no caer en la lectura como decodificación y los tristes resultados en la comprensión lectora que esto depara. Pero no comprendí por qué la concepción de la adquisición de un código no aparecía en la norma curricular articulada con la de la representación. Por el contrario, se omitía todo abordaje analítico del sistema.

Estas cuestiones deben ser tan familiares para los maestros de primer grado como lo era para mí el hecho de que en ninguna parte del diseño de segundo ciclo apareciera el reconocimiento del territorio argentino en los mapas, sus límites y determinantes del clima, los principales ríos y relieves, antes de analizar paisajes y problemas ambientales de lo más interesantes que plantean las propuestas de desarrollo curricular. O tampoco resulta una novedad el hecho de que al final del primer ciclo, cuando los chicos tienen entre 8 y 9 años, el uso del "repertorio de operaciones conocidas" de los que prescribe el diseño, requiera de un trabajo explícito, graduado y sistemático con la memoria para cada una de las innombradas "tablas de multiplicar" durante meses, a fin de evitarles largas reflexiones sumativas en la resolución de operaciones que se les presentarán en el segundo ciclo.

Lo cierto es que, para mí, la omisión de la perspectiva de la alfabetización como adquisición de un código, en los materiales curriculares para primer grado, generó una experiencia algo inesperada.

Mientras trabajábamos con el nombre propio, incorporé sin dudar ejercicios de reconocimiento de las vocales en la inicial de distintas palabras, en los nombres propios y en el vocabulario de cuentos y poesías. Aunque no estaba en el diseño, las vocales se me presentaron con tanta contundencia que las presenté con carteles de colores y distinto tipo de ejercicios pensando que la normativa las daba por entendido.

Semanas más tarde, con el mismo supuesto, decidí trabajar las vocales con la canción "La mar estaba serena", a fin de extraer las sílabas LA, LE, LI, LO, LU y MA, ME, MI, MO, MU. Seguidamente, con la canción del ratón que encontró Martín debajo de un botón, me propuse obtener las sílabas TO y TI y extender a TA, TE, TU. Así que llegamos un día, después de mucho leer, cantar y reconocer palabras y letras, a la escena del pizarrón con todas las sílabas encolumnadas y los chicos proponiendo palabras a partir de combinarlas. Cuando armaban una palabra, no podían dejar de sorprenderse y venir a contarme con los ojos muy grandes: "¡seño! ...ma...ti... ¡Mati soy yo! Es mi nombre”, “¡seño! ma... ma, ¡mamá!”, y así con Lola, loma, mula, lame, malo, toma y Milo, entre otras.

Lo comenté con mi querida paralela y guía, una maestra que lleva varios años en primer grado y a quien sigo en la planificación de proyectos, secuencias y actividades habituales. Estábamos en el hall de entrada y yo tranquilamente iba diciendo "ma, me, mi..." cuando solamente me tomó del brazo. Y ahí mismo me di cuenta de tres cosas:

a) que esa conversación tendríamos que haberla tenido a solas en el aula de ella o en la mía;

b) que efectivamente, en el diseño no aparecía un trabajo graduado de este tipo porque estaba contraindicado, no porque fuera obvio y hubiera que hacerlo de cualquier modo;

c) que en la escuela primaria la alfabetización es concebida oficialmente como construcción de un sistema de representación al que se accede mediante prácticas con sentido social y no como adquisición de un código al que se accede analíticamente, partiendo en partes, uniendo y entrenando.

Luego mi compañera me contó que el año anterior, en el contexto de aislamiento por COVID habían acordado con el maestro que estaba en mi grado, trabajar de esa manera en que yo lo hice, agregando incluso a "Susana" con la "sal", la "masa", el permiso para "salir sola" y otras expresiones por el estilo, porque la falta de una experiencia cotidiana escolar había estado impidiendo que los chicos llegasen espontáneamente o por su propia actividad a la hipótesis alfabética. Y que recién entonces algunos habían empezado a mostrarse más seguros y habían empezado a escribir algunas palabras. A este importante gesto de confianza respondí con gran reconocimiento por la decisión que habían tomado. Pero la conversación terminó con la advertencia "sin embargo, este año, viene la capacitadora de prácticas del lenguaje, jasí que olvidate!... a lo sumo hacelo oralmente pero no lo escribas y que no les quede a ellos nada en el cuaderno". Este aviso me pareció tan desafiante y valiente como el de una compañera de hace muchos años que me había contado que cuando nadie la miraba, les tomaba la manito a los chicos con el lápiz para hacer con ellos el trazo de las letras.

Me quedé preocupada por mi ingenuidad. Me imaginé tres escenas con la misma y lamentable conclusión.

a) Un niño lleva el cuadernito a casa y la mamá que también es maestra lo mira y se encuentra con esa tarea. Piensa "Vale no se siente cómoda con las nuevas perspectivas de la alfabetización". Y arriba a la sentencia "Es una señora grande y estuvo mucho tiempo fuera de la escuela".

b) La vicedirectora mira mi carpeta didáctica y encuentra esta actividad. Piensa “¡qué bueno que este año tendrán capacitación en prácticas del lenguaje porque Vale la necesita!... Es una señora grande y estuvo mucho tiempo fuera de la escuela"

c) La capacitadora me habla durante horas de las nuevas modalidades que ya he venido leyendo por años. En un momento me desahogo preguntándole iY cómo esperan que tengamos diálogos individualizados de ese tipo en un aula de primer grado? Y la capacitadora piensa, "claro...Es una señora grande y estuvo mucho tiempo fuera de la escuela".

Lo que me preocupó no fue tanto echar por tierra horas de tintura y maquillaje, sino el riesgo de ser colocada "afuera" del 
horizonte de mutuo entendimiento entre pares, capacitadores y directivos por hacer algo que sólo podía hacerse de manera marginal, en la excepcionalidad del aislamiento preventivo o como conversación de aula sin dejar registro. El fantasma del maestro autoritario me advirtió que estaba asomándome a un límite.

En algún texto de psicología laboral leí que el oficio, el arte del trabajador experimentado, tiene que ver en gran parte con manejarse cómodamente en el terreno de imposibilidad que plantea el sistema normativo, haciendo a pesar de esto, su tarea. Es decir, a partir del saber de experiencia (Contreras, 2011) logran tomar atajos y deslizarse entre las normas para hacer su obra. Este, tal vez, sea el núcleo del saber de esas maestras de las que se dice, "tiene mucha experiencia" y a las que tanto admiro. La creación didáctica que aparecía en las lecturas de mi formación inicial con signo negativo, puede tener a veces el signo positivo de la productividad y del logro. Es lo que se consigue a costo de cumplir incumpliendo ciertos contenidos no tan consensuados, ciertos saberes que son terreno de disputa entre teorías que no logran articularse. No estoy hablando de caer en la explicación condescendiente que descree de las inteligencias de los estudiantes y en su capacidad de construir el sistema por sí mismos, ni de arrojarse a una serie interminable de renglones para practicar las grafías, ni de restringir el vocabulario sólo a las palabras que tienen determinadas letras. Esto sería retroceder a enfoques y estrategias obsoletas de alfabetización que la investigación ha demostrado ineficaces para la apropiación de la lengua escrita. Me refiero, por el contrario, a la astucia para encontrar el momento y el modo oportuno de facilitar el aprendizaje recurriendo a un gesto o a una modalidad de intervención traída de aquel modelo descartado, sin que esto deje rastros, sin afectar la orientación del encuadre curricular. Por eso creo que es creación enseñar siguiendo el diseño curricular, las intuiciones nombradas con la "voz de la experiencia", las dudas, el deseo y otros oráculos.

Como me decías hace poco comentando este artículo, la creación no desiste de lo dado, lo toma y lo recrea. La creación siempre es apropiación y versión singular, más que mera oposición. $Y$ por eso creo que se requiere de mucha experiencia para ser un maestro creativo porque ser creativo en la escuela, es, en definitiva, enseñar...Y lo que sigue puede verse como provocación, pero debería contextualizarse en la experiencia que acabo de narrar, en lo que aprendí de mis pares: digo que ser creativo tal vez tenga que ver con enseñar, a pesar del diseño curricular, pero sin salirse de él. Y remarco "a pesar" y "sin salirse" porque el diseño curricular es también un acuerdo entre el Estado y la sociedad sobre lo que ha de enseñar la escuela, con toda una estructura documental y de personal especializado en su difusión. Entonces en respuesta a tu pregunta sobre mis prácticas y la creatividad dejaría suspendida esta expresión sobre la creatividad como práctica de enseñar "a pesar y sin salirse del diseño curricular" con la intención de resolverla en conversación con tu relato.

\section{Aproximaciones al currículum de un investigador educativo}

Para contarte de qué manera pienso o pensaba el currículum voy a traer al texto algunos momentos de mi historia de acercamientos y alejamientos al tema. Mis primeras aproximaciones al debate sobre el currículum se iniciaron por los primeros años de la década de 1990, pero más como investigador educativo que por entonces hacía etnografía, que como docente. Quería estudiar en profundidad lo que llamaba "currículum en acción" (Suárez, 1995): los modos en que los sujetos de la acción pedagógica cargan de sentido localmente a sus prácticas de enseñanza al mismo tiempo que recepcionan, resignifican y recrean las normas curri- culares. Esa investigación pretendía salir de una controversia, a mi entender poco productiva, en el campo del currículum entre los que lo entendían tan solo como prescripción, proyecto, aspiración, norma o patrón, y sus versiones de uso: “documento oficial", "diseño curricular", y aquellos que proponían nociones un tanto más imprecisas de currículum como práctica, perfomance o experiencia vivida, y sus variaciones como "currículum oculto" y "currículum real". Para escapar de la trampa, experimentaba con la fórmula con la que Stenhouse pretendía zanjar esta polaridad: currículum = prescripción + práctica, en la que ya venía profundizando, entre otros, Goodson (2000). Por eso, hice mía la advertencia de entender al currículum como "el medio con el cual se hace públicamente disponible la experiencia consistente en intentar poner en práctica una propuesta educativa" (Stenhouse, 1991, p.30). Es decir, desde mi perspectiva de investigación, me inclinaba a aproximarme al currículum como una vía para la composición y la disposición pública de una obra que da cuenta de la experiencia (siempre creativa) de poner en práctica (siempre de manera situada) una propuesta educativa. Pensar el currículum como investigación de la experiencia de enseñar, era la pista a seguir.

Para acercarme a la "construcción local del currículum", entonces, pretendía comprender cómo los actores escolares necesariamente recepcionan, interpretan, se apropian y le dan un nuevo significado -esto es re-crean- a la narrativa oficial del currículum y su mandato referido a la transmisión escolar, de acuerdo con una lógica de localización más que de concreción o actualización en la práctica. La noción de localización me permitía resaltar el carácter local, situado, de toda recepción, y eludir la ilusión tecnocrática de un control exhaustivo de la prescripción curricular central sobre la práctica de enseñanza periférica. Pero también me orientaba para dar cuenta de cómo esa creación localizada está sujeta a influencias, tradiciones y sentidos heredados o impuestos administrativamente. La creación, o la imaginación, o la innovación semántica, como ya te comenté, no se producen en el aire y según alguna lógica caprichosa, sino en relación con un pasado prefigurado que se hace presente como tradición: tradición e innovación se encuentran más enlazadas de lo que se sospecha.

Quería estudiar etnográficamente el currículum como el territorio y el medio de una experiencia creativa y como interpretación creativa de esa experiencia. Algo así como propone Giddens mediante la noción de "doble hermenéutica" (1995): el currículum como interpretación de las interpretaciones que ponen a jugar creativamente los sujetos escolares cuando hacen la enseñanza y localizan el currículum; cuando obran en la escuela, resuelven mediante sus astucias y "tácticas" los problemas de la vida cotidiana y resisten y tergiversan las "estrategias" del poder (De Certeau, 2007). Llegué así a la comprensión del currículum re-creado por los docentes en la escuela como una forma de "conocimiento local" (Geertz, 1994) que requiere de una descripción densa y narrativa para indagar en él y disponerlo públicamente, de un registro narrativo que documentara lo no documentado del obrar pedagógico de los docentes cuando hacen la enseñanza. Para eso resultaba conveniente una larga estancia en las escuelas, entablar con sus habitantes relaciones de confianza y empatía y solicitarles historias, conversar con ellos, y escribir mi propia obra, el relato etnográfico: una creación que daba cuenta de mi interpretación de esas otras creaciones que mis sujetos de investigación, fundamentalmente los docentes, me confiaban en sus entrevistas, documentos personales y textos.

Cabe advertirte precisamente aquí, que ese trabajo de campo y esa producción escritural estaban reguladas por los criterios metodológicos y las reglas de composición de la antropología de la educación, una lengua extranjera diferente a la de los docentes 
y cuyo hablante y traductor era yo. De esta manera, las sutiles creaciones de los docentes en el currículum, esas mínimas alteraciones locales que supone cualquier lectura o recepción y las singulares y situadas comprensiones pedagógicas que las informan, reelaboradas, reinterpretadas y ficcionalizadas como relatos de experiencia o historias de sí, como las que acabás de contarme, quedaban relegadas al anexo de la etnografía, y sus autores, al anonimato. O bien eran traídas al cuerpo mi reporte etnográfico como referencias o transcripciones de fuentes, también anónimas, para soportar e informar mi propia interpretación.

Otro de mis acercamientos al currículum fue desde el ministerio de educación de la nación, a comienzos del siglo, como uno de los coordinadores del proyecto "El currículum en el aula". En esta oportunidad, junto a un equipo, nos animamos a reconstruir el currículum en acción mediante la elaboración escrita de relatos de experiencia en colectivos de docentes. Queríamos que esas historias de escuela, esos relatos que narran cómo los docentes crean en la enseñanza, fueran "oficializados" en documentos curriculares dispuestos públicamente por el ministerio y mostraran la construcción cotidiana del currículum escolar autorizando a los docentes narradores como sujetos creadores de "obras de enseñanza" (Alliaud, 2011, 2017). Otra vez, mi aproximación al problema no era como docente, sino como agente o funcionario del estado y como parte del plantel que debía traducir orientaciones de política en dispositivos de intervención en el campo del currículum. Desde ese lugar, frente a la indolencia tecnocrática de la reforma educativa de los 90 y como respuesta a su pragmática descalificante de los saberes y experiencias de los docentes en la "implementación del currículum" (Suárez, 2004), pretendimos reconocer y recuperar las palabras y tramas que los docentes crean para atribuir significados pedagógicos a sus mundos escolares. Mediante un dispositivo metodológico que regulaba la indagación narrativa de la propia experiencia, esperábamos que fueran los propios docentes participantes los que interpretaran y re-interpretaran sus experiencias de "hacer el currículum" y los que crearan y re-crearan discursos, comprensiones y saberes alrededor de ellas. También, y esto era lo más disruptivo, pretendíamos que ratificaran con su firma y autoría la creatividad e imaginación pedagógica que despliegan en el desarrollo de su oficio como maestros y profesores.

Llamamos a ese dispositivo "documentación de experiencias pedagógicas" (Suárez y Brito, 2001). Y junto con los ministerios, equipos técnicos y docentes de quince provincias, pudimos llevarlo adelante a través de una red de 192 coordinadores, que eran docentes que participaban en un trayecto de formación, acompañamiento y asesoramiento metodológico. La experiencia inicial fue entre 2000 y 2001, y sin bien se interrumpió por la crisis económica, social, política e institucional que sufrió el país, pudimos retomarla en 2007 y 2008 mediante un convenio entre el Laboratorio de Políticas Públicas de Buenos Aires (donde trabajaba por entonces) y el Ministerio de Educación. En esa oportunidad logramos publicar alrededor de mil relatos de experiencia escritos por docentes, e involucrar muchos educadores de todo el país en la documentación narrativa de sus experiencias de escuela.

Lo curioso de aquella movida fue la instantánea desconfianza que provocó en los docentes nuestra invitación ministerial a que narraran "alguna experiencia escolar que por algún motivo" consideran como "pedagógicamente significativa". A eso se sumó el desconcierto que suscitó que les pidiéramos que leyeran sus borradores en pequeños colectivos coordinados por otro docente y que luego conversaran en torno de los relatos y sus lecturas. Y también, el fastidio que mostraron cuando les solicitamos que volvieran a escribir la historia una y otra vez, revi- sitando la experiencia e indagando en las palabras y las tramas narrativas que componían, a partir de esa rueda espiralada de diálogos y versiones de relatos. No estaban acostumbrados a escribir palabras que no se inscribieran en el aparato de información, evaluación y control que la reforma había consolidado, sobre todo cuando eran requeridas por el gobierno educativo, la administración escolar y sus equipos tecno burocráticos. Por entonces, nadie les preguntaba nada que no estuviera asociado con esa matriz jerárquica que reducía su experiencia, su saber y su discurso como objeto de intervención. También fue llamativo constatar cómo la gramática curricular disciplinar y especializada de la reforma, persistente e instalada en el organigrama del ministerio, seguía traccionando hacia el centro el flujo de la creación en el campo del currículum (Suárez, 2003) e intentando capturar la singularidad e integralidad de la experiencia en las clasificaciones externas y vicarias del currículum oficial. Lo cierto es que, a partir de esa experiencia en el territorio de las políticas curriculares, de ese primer y masivo intento por documentar la creativa construcción escolar del currículum, comencé a pensar la posibilidad de estudiar las narrativas autobiográficas como vía, metodología y soporte para la producción de saber pedagógico. Era una oportunidad para indagar y experimentar en territorio las potencialidades del dispositivo de trabajo que habíamos creado y, en cierta medida, improvisado.

Luego de la constatación de la raigambre y resonancia tecnocrática del significante currículum en la arquitectura institucional del mundo escolar y en la jerga pedagógica de los docentes, fui abandonando de a poco la retórica del currículum. La palabra currículum había llegado a la Argentina en la década del '60 inscripta en los documentos técnicos y recomendaciones de los organismos internacionales del mundo económico (OCDE) que orientaron la reforma educativa tecnocrática, conservadora y autoritaria de la dictadura de Onganía, y había renovado su sentido en el discurso y las prácticas políticas de la reforma educativa neoliberal de la década de los '90, también de la mano de agencias internacionales de crédito (Banco Mundial, BID). Y en serie con esos anclajes discursivos, el currículum entretejió su tradición, su retórica y su lenguaje con las políticas de conocimiento educativo que despojan a los docentes de la posibilidad de crear sus obras pedagógicas y los convierten en el objeto de sus operaciones. Más acá de esa tradición colonial, por su parte, los maestros y profesores no mencionan en sus relatos al currículum sino sólo para referirse al "diseño curricular", al documento oficial que señala y pretende regular, secuenciar y clasificar sus prácticas pedagógicas. $\mathrm{O}$ bien, se refieren a éste para mencionar a un cierto mandato normativo vertical, centralizado, y portador del saber disciplinar y didáctico especializado.

Tal como insinuás en tu historia, el suelo de la creatividad e imaginación pedagógica de los docentes parece extenderse en el espacio y el tiempo a donde no llega la prescripción curricular, aun cuando ésta sea una referencia ineludible y pretenda abarcar completamente el territorio de las prácticas de enseñanza. Con "suelo" quiero referirme a una experiencia del espacio, del tiempo y de la presencia con los otros en la cotidianeidad de la escuela $y$, si se quiere, en el currículum. Una experiencia que reclama un relato, una historia, pero que también requiere de un receptor, un escucha, un lector, un par interlocutor, una comunidad de interpretación, para constituirse en saber de oficio, en obra pedagógica, en creatividad documentada. Por eso, todo mi trabajo de investigar la creatividad pedagógica de los docentes se orientó más bien al campo de la narrativa pedagógica, la investigación-formación-acción docente y las redes de colaboración e investigación coparticipada. Durante los últimos veinte años, en la Universidad de Buenos Aires, venimos estudiando y experi- 
mentando, junto con docentes organizados en colectivos y redes, la "documentación narrativa de experiencias pedagógicas" como una modalidad de investigación que pretende dar cuenta de la experiencia escolar y de las interpretaciones que ponen a jugar los docentes cuando la hacen y narran (Suárez, 2007, 2017). Y allí, la serie que se arma es otra: experiencia escolar, saber de oficio, narrativa pedagógica, relatos de experiencia, saber pedagógico, obra pedagógica. Los relatos que escriben y reescriben los docentes en una suerte de espiral hermenéutica colectiva de lecturas, comentarios y conversaciones entre pares, dispara y secuencia la indagación del narrador. Son creaciones pedagógicas, obras que dan cuenta de una investigación y que tornan públicamente disponible la experiencia de llevar a la práctica una propuesta educativa.

\section{Conversación para concluir}

Valeria M: - Por lo general, un artículo que plantea una problemática, un enfoque o un concepto, suele cerrarse de manera más o menos pretenciosa con el término conclusión. El desarrollo presentado en el texto concluye, afirma y al final se detiene a puntualizar los aspectos a resaltar, las enseñanzas que ofrece, lo que queda seguro. Siempre me pareció demasiado ambiciosa y solemne esa designación tan afirmativa. Sin embargo, creo que la cuestión del currículum que estamos conversando, esa que lo entiende como creación y propone que el currículum se realiza, se proyecta y se experimenta, justifica alguna conclusión, algo sobre lo cual afirmarnos.

Daniel S: - Puede ser... pero prefiero el tono dubitativo, incierto, inseguro, del que está conversando, del que se está enfrentando consigo mismo, con sus palabras e ideas cuando conversa con otro. Me inclino últimamente, y sobre todo luego de este intercambio con vos, a terminar este ensayo mediante un diálogo que satisfaga la expectativa formal de la conclusión, pero que permita prolongar la inquietud que me provocó la conversación... Tal vez lo más interesante de lo que dialogamos sean los detalles y secretos de la experiencia de enseñar y de investigar la enseñanza, o de volver a la escuela, o de abandonar un lenguaje. Tal vez lo que podamos concluir sea que, al menos en nuestra experiencia, el campo del currículum es un terreno resbaladizo y sinuoso, difícil y muy connotado, pero inevitable.

Valeria M: - Leyendo en tu relato, las sucesivas incursiones en la problemática del currículum y las respuestas de los docentes que comentás, me veo reflejada en un profesorado "hablado" por la reforma de los 90. La cantidad y variedad de acciones de la política curricular federal nos interpeló de tal forma que apropiarse del discurso de la reforma fue central para desempeñarnos y comunicarnos en un sistema que se reconfiguraba. Así también, cuando me refiero a lo curricular, como los docentes que recordás en tu relato, sólo tengo en mente la prescripción a la que llamo diseño para referirme tanto al documento de base como a los desarrollos posteriores. Todo lo demás corresponde a las prácticas y al terreno de la experiencia escolar, de la que a veces nos toca dar cuenta a la luz del diseño y otras veces podemos transitar sin más. Dejar de lado esa posición de maestra que responde al currículum (entendido como prescripción) y tomar la posición de tesista, narradora e investigadora me abrió la posibilidad de ensayar una búsqueda de la creatividad y de lo que aprendí sobre la creatividad docente, a través de mi propia experiencia.

Daniel S: - Pero también, como tesista, narradora e investigadora, te sugiero retomar la perspectiva de la maestra que vuelve a la escuela luego de un recorrido profesional y vital significativo, luego de ese "bucle biográfico" que narrás, y que vive la escuela y el encuentro pedagógico con los niños como un punto de llegada, o como el retorno a algo que nunca se había vivido. Los secretos, misterios y creaciones pedagógicas en torno de la alfabetización escolar narrados como experiencia autobiográfica condensan esa multiplicidad de perspectivas (tesista, narradora, investigadora y maestra). Por eso me parece sugerente volver a pensar ese ejercicio de biografización, de autoetnografía y de escritura como posición de enunciación y tono para la elaboración de relatos de experiencia que den cuenta de la creatividad del docente en el currículum. Tal vez las narrativas de sí, las historias de escuela, las confesiones de aula, los relatos de experiencia, entendidos como obras pedagógicas escritas por docentes, como producciones originales de saber pedagógico situado, puedan ofrecernos indicios de la creatividad de los docentes en el currículum, pero también, y de manera decisiva, puedan ayudar a revitalizar el lenguaje de la educación, a estimular la conversación pedagógica y a recrear ese territorio de saberes, experiencias, discursos y sujetos que viene siendo la pedagogía.

Valeria M: - Quisiera resaltar esta idea que proponés, la de la creatividad de los docentes en el currículum. En mi relato, dejé abierta una expresión sobre la creatividad docente como aquella disposición a enseñar "a pesar" del diseño curricular, pero "sin salirse" de éste, a riesgo de enfrentarse con la imagen temida del autoritarismo. Pero creo que tu manera de expresar la creatividad como posibilidad en el currículum profundiza esa expresión y la torna más fértil. Al referirme, como otros docentes, a lo curricular como lo prescripto en donde se impone el lenguaje de las didácticas específicas y queda silenciado el saber y hacer de los maestros, la creatividad queda en cuestión. Ya sea como promotora de contenidos mal acuñados (las "creaciones didácticas"), o bien como salida productiva y positiva que resiste a lo dispuesto verticalmente, aquello que producimos los maestros es de alguna manera inevitable.

No obstante, como anticipás, el diseño curricular expresa un consenso. En su texto, como veíamos, se puede vislumbrar, por omisión, el conflicto entre perspectivas en pugna. Pero considero que se puede constatar que han logrado imponerse, hasta el momento, tal vez a costa de censurar masivamente cualquier heterodoxia, aquellas perspectivas que vienen siendo más respetuosas de la inteligencia y la afectividad infantil. No considero creativo oponerme al diseño curricular. Esto sería salir del territorio común de los maestros, correrse de lo compartido y alentar propuestas que en los debates disciplinares han resultado superadas. En este sentido, el diseño es en sí mismo una propuesta a desarrollar. El concepto de currículum como desarrollo, como investigación y como territorio para la experiencia pedagógica, permite ver las cosas de otro modo y la idea de creatividad en el currículum sintetiza bien esta posibilidad. Cuando dije "sin salirse" de él, me refería no tanto al texto escrito que cristaliza cierto momento del debate, sino al currículum entendido como territorio y como un medio, tal como sugerís. La creatividad docente en el currículum tiene que ver, entonces, con la capacidad de sostener un lenguaje común como condición de posibilidad para disponerse en conversación con lo imposible y a su vez con lo promisorio de la experiencia de enseñar.

\section{Referencias bibliográficas}

Alliaud, A. (2004). La escuela y los docentes. ¿Eterno retorno o permanencia constante? Cuaderno de Pedagogía, 12(VII), 91105.

Alliaud, A. (2011). Los maestros y sus obras. Revista Educación y pedagogía, 61(23), 81-92

Alliaud, A. (2017). Los artesanos de la enseñanza. Paidós. 
Braslavsky, B. (2014 [1962]). La querella de los métodos en la enseñanza de la lectura. Unipe.

Camilloni, A. (1996). Corrientes didácticas contemporáneas. Paidós.

Cucuzza, H. R. (dir.) y Spregelburd, R. (codir.) (2012). Historia de la Lectura y la Escritura en la Argentina. Del catecismo colonial a las netbooks estatales. Calderón.

Davini, M. (2014). La formación en la práctica docente. Paidós.

De Certeau, M. (2007). La invención de lo cotidiano. Universidad Iberoamericana.

Ferreiro, E. (1997). Alfabetización. Teoría y práctica. Siglo XXI Editores.

Geertz, C. (1994). Conocimiento local. Paidós.

Giddens, A. (1995). La constitución de la sociedad. Amorrortu.

Goodson, I. (2000). El cambio en el currículum. Octaedro.

Litwin, E. (1997). Las configuraciones didácticas. Paidós.

Pujato, B. (2009). El ABC de la alfabetización. Homo Sapiens.

Ricoeur, P. (2004). Del texto a la acción. Fondo de Cultura Económica.
Stenhouse, L. (1991). Investigación y desarrollo del currículum. Paidós.

Suárez, D. (1995). Formación docente, currículum e identidad. Revista Argentina de Educación (RAE), 22, 35-52.

Suárez, D. (2003). Dispersión curricular, descalificación docente y medición de lo obvio. Revista Novedades Educativas, 155(15), 23-27.

Suárez, D. (2007). Docentes, narrativa e investigación educativa. En Sverdlick, I. (comp.), La investigación educativa. Una herramienta de conocimiento y acción. Novedades Educativas.

Suárez, D. (2017). Docentes, relatos de experiencia y saberes pedagógicos. La documentación narrativa de experiencias en la escuela. Revista de Investigación Cualitativa 2(1), 42-54. DOI: http://dx.doi.org/10.23935/2016/01034

Suárez, D., Brito, A. (2001). Documentar la enseñanza. Revista El Monitor, 4, 26-27.

Suárez, D., Metzdorff, V. (2018). Narrar la experiencia educativa como formación. Revista Espacios en Blanco, 28, 49-74. 